\title{
Synthetic sugar cassettes for the efficient production of flavonol glycosides in Escherichia coli
}

\author{
Prakash Parajuli, Ramesh Prasad Pandey, Nguyen Thi Huyen Trang, Amit Kumar Chaudhary \\ and Jae Kyung Sohng*
}

\begin{abstract}
Background: A multi-monocistronic synthetic vector was used to assemble multiple genes of a nucleotide diphosphate (NDP)-sugar biosynthetic pathway to construct robust genetic circuits for the production of valuable flavonoid glycosides in Escherichia coli. Characterized functional genes involved in the biosynthesis of uridine diphosphate (UDP)-glucose and thymidine diphosphate (TDP)-rhamnose from various microbial sources along with glucose facilitator diffusion protein $(\mathrm{g} / \mathrm{f})$ and glucokinase $(\mathrm{g} / \mathrm{k})$ from Zymomonas mobilis were assembled and overexpressed in a single synthetic multi-monocistronic operon.

Results: The newly generated NDP-sugars biosynthesis circuits along with regiospecific glycosyltransferases from plants were introduced in E. coli BL21 (DE3) to probe the bioconversion of fisetin, a medicinally important polyphenol produced by various plants. As a result, approximately $1.178 \mathrm{~g}$ of fisetin 3-O-glucoside and $1.026 \mathrm{~g}$ of fisetin 3-O-rhamnoside were produced in UDP-glucose and TDP-rhamnose biosynthesis systems respectively, after $48 \mathrm{~h}$ of incubation in $3 \mathrm{~L}$ fermentor while supplementing $0.9 \mathrm{~g}$ of fisetin. These yields of fisetin glycosides represent $\sim 99 \%$ of bioconversion of exogenously supplemented fisetin. The systems were also found to be highly effective in bio-transforming other flavonols (quercetin, kaempferol, myricetin) into their respective glycosides, achieving over $95 \%$ substrate conversion.

Conclusion: The construction of a synthetic expression vector for bacterial cell factory followed by subsequent redirection of metabolic flux towards desirable products have always been revolutionized the biotechnological processes and technologies. This multi-monocistronic synthetic vector in a microbial platform is customizable to defined task and would certainly be useful for applications in producing and modifying such therapeutically valued plant secondary metabolites.
\end{abstract}

Keywords: NDP-sugars biosynthesis circuits, Glycosyltransferase, Multi-monocistronic vector, Flavonol glycosides

\section{Background}

The bioactive flavonoids are considered as the most important phytochemicals abundantly present in daily dietary foods and are of great interest due to their diverse health beneficial activities for the management of chronic diseases [1]. Flavonols are a class of flavonoids having 3-hydroxyflavone backbone and different stems

\footnotetext{
*Correspondence: sohng@sunmoon.ac.kr

Department of BT-Convergent Pharmaceutical Engineering, Institute of Biomolecule Reconstruction, Sun Moon University, 70 Sunmoon-ro 221, Tangjeong-myeon, Asan-Si, Chungnam 336-708, Republic of Korea
}

hydroxylated by phenolic -OH groups to characterize as quercetin, kaempferol, myricetin, morin and fisetin [2]. Being the most active compounds within flavonoid sub-group, they exhibit wide range of biological activities such as prevention of cardiovascular diseases [3, 4], antioxidant [5], anti-diabetic activity [6], anticancer activity [7], etc. Although flavonols are virtually present in almost all the vegetables and fruits, they are usually present in diverse glycoside forms with considerable amounts in our normal diet [3]. Glycosides are produced through an approach called glycosylation which is considered 
to be a key modification in plant secondary metabolites and it is also one of the major factor that determines natural products solubility, bioactivity and bioavailability [8]. Additionally, most flavonoid drugs (quercetin 3-O-rutinoside, daidzein 8-C-glucoside) presently in clinical applications are in the form of glycosides [1]. So these significance have attracted considerable interest in current research trend to produce them rationally in industrial scale. Microbial biotransformation is one of the biotechnological approach for the production of such bioactive flavonoids and their glycosides in desirable scale $[1,9]$. Biotransformation of flavonoids could be achieved from many microorganisms including species of Aspergillus, Bacillus, Saccharomyces, Streptomyces, Escherichia coli $[1,10]$.

Escherichia coli is one of the industrially important microorganisms, widely used in different research facilities for the production of therapeutic proteins [11], biobased chemicals and bio-fuel production [12], production and modifications of natural products [13, 14], production of valuable therapeutics and cosmetics [15-18], neutraceuticals $[18,19]$, etc. $E$. coli is often possible to grow in high cell densities by utilizing simple carbon sources and dissolve oxygen to accomplish volumetric productivity [20]. Additionally, recently developed synthetic biology approaches helped to establish $E$. coli based efficient cell factories by genetic manipulations or recombining foreign genetic materials for efficient utilization of simple precursors with high growth rate, avoiding metabolic shutdown [21].

In this aspect, several flavonols have been biotransformed to produce their glycosides using different metabolic or combinatorial approaches and synthesized compounds with high stereo- and regio-selectively [22]. Overexpression of a single glycosyltransferase in wild type host strain and whole-cell biotransformation of flavonoids to glycosides would be an easy approach. But, construction of a cell factory through synthetic approaches (enzyme engineering, knockdown and knockout for metabolic flux control, heterologous expression of pathway specific genes) are the key parameters basically involved while designing high yield production strains [23]. Application of synthetic vector and heterologous expression of multiple genes into single vector has been one of the alternatives while talking about an efficient cell factory construction and synthesis of modified secondary metabolites [24, 25]. Additionally, metabolic engineering approaches have been applied by various groups to synthesize diverse glycosides of flavonols using E. coli biotransformation systems.

In the current experiment, the multi-monocistronic vector piBR181 [26] was used as a vehicle to construct the sugar cassettes and employ them for the glycosylation of flavonols. For the production of high value flavonoid glycosides, nucleotide diphosphate (NDP)-sugar biosynthesis specific genes were chosen and assembled in a single vector along with glucose facilitator diffusion proteins (glf and glk) from Zymomonas mobilis [27, 28]. To facilitate the synthesis of flavonol glucosides and rhamnosides, regiospecific glycosyltransferases (GTs), specifically, uridine diphosphate (UDP)-3-O-glycosyltransferase (UGT78K1) from Glycine max and flavonols-3-O-rhamnosyltransferase (ArGt-3) from Arabidopsis thaliana were selected. Additionally, consumption of glucose as the sole carbon source was optimized in the E. coli strain and its subsequent utilization by the facilitator proteins for accelerating the production of glycosides was studied. These single vector NDP-sugar biosynthesis systems were compared with previously reported multi vector biotransformation systems [29-31] for increased titer of flavonoid glycosides under identical conditions.

\section{Results and discussion}

\section{Construction of NDP-sugar biosynthesis systems}

NDP-sugar biosynthesis genes from different source organisms were individually cloned and assembled to construct UDP-glucose and thymidine diphosphate (TDP)-rhamnose sugar cassettes in piBR181 vector containing multi-monocistronic operon systems [26]. Five different UDP-glucose pathway genes including glucose facilitator diffusion protein and flavonol-3-O-glycosyltransferase were cloned successfully to construct a UDPglucose biosynthesis system. Seven TDP-L-rhamnose biosynthesis genes, including facilitator diffusion protein, were cloned and assembled to construct TDP-rhamnose sugar cassettes (Figure 1). Sources and cloning strategies for each gene are explained in materials and methods. The vector physical map of both sugar cassettes and assembly of genes are given in supplementary data (Additional file 1: Figures S1, S2).

The UDP-glucose system consists of three UDPglucose biosynthesis genes: glucokinase (glk; PCR size $984 \mathrm{~kb})$ which catalyzes the addition of phosphate groups into the 6-hydroxyl position of D-glucose immediately after entry into the cell, phosphoglucomutase (pgm2; 1,749 kb) which synthesizes glucose 1-phosphate from glucose-6-phosphate, and glucose 1-phosphate uridylyltransferase (gall; $908 \mathrm{~kb}$ ) which transfers the uridine diphosphate (UDP) group to make UDPglucose. In addition to the above three genes, glucose facilitator diffusion protein $(g l f ; 1,422 \mathrm{~kb})$, which helps in the internalization of extracellular glucose present in the medium into the cell to increase the pool of UDPglucose and the regiospecific flavonol 3-O-glycosyltransferase (UGT78K1; 1,344 bp), which catalyzes the transfer of glucose moiety from activated UDP-glucose 


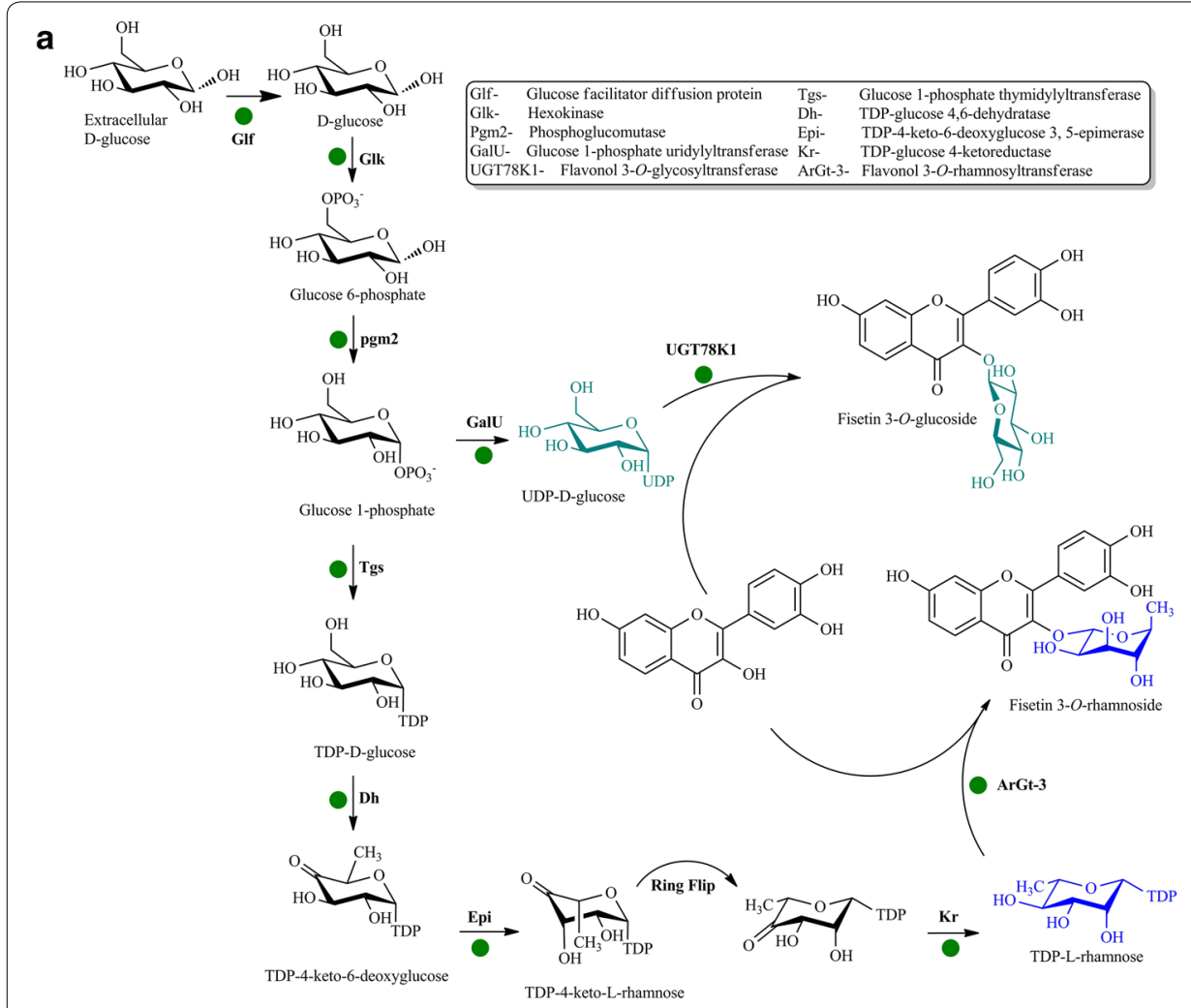

b

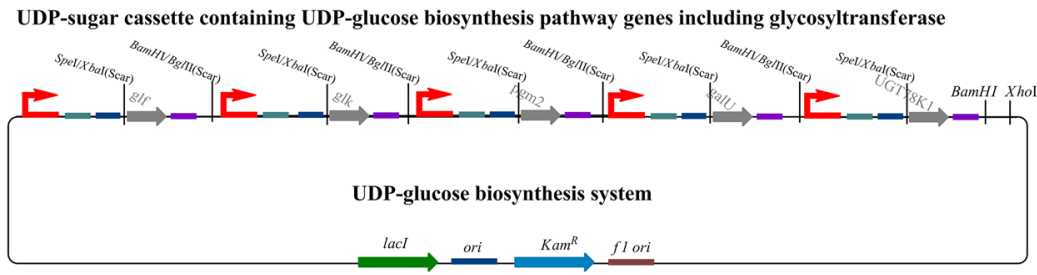

Deoxy sugar cassette containing TDP-rhamnose biosynthesis pathway genes and pET32(a)+ ArGt-3 under same promoter (T7)

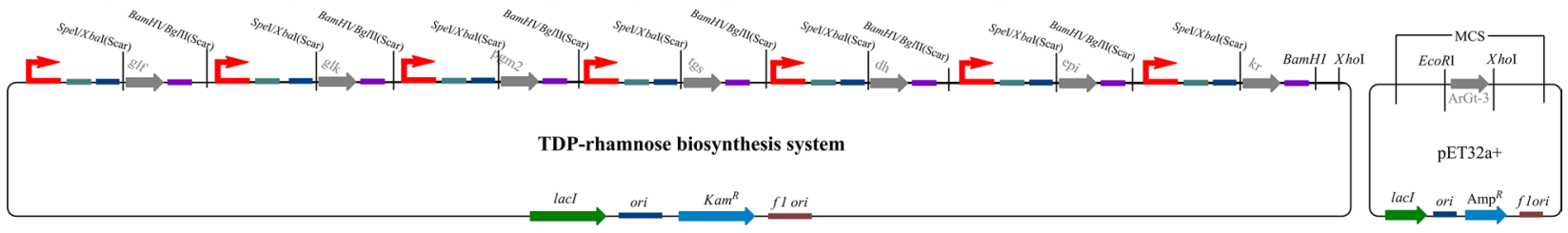

Figure 1 a NDP-sugar (UDP-D-glucose and TDP-L-rhamnose) biosynthesis pathway in E. coli showing the pathway overexpressed genes and glycosyltransferases (green dots). b Newly constructed sugar cassettes for the biosynthesis of TDP-rhamnose and UDP-glucose using a multi-monocistronic vector piBR181.

into the acceptor flavonols at the 3-hydroxyl position, were also assembled in the same vector to generate a 7,312 bp UDP-glucose system. This UDP-glucose cassette was transferred into E. coli BL21 (DE3) for further biotransformation experiments. Similarly, the same $g l f$, $g l k$, and $p g m 2$ genes were recombined along with glucose 1-phosphate thymidylyltransferase (tgs; $882 \mathrm{bp}$, synthesizes TDP-glucose from glucose-1-phosphate and thymidine triphosphate), and TDP-glucose 4, 6-dehydratase (dh; 1,086 bp) to make TDP-4-keto-6-deoxyglucose intermediate. The modifying genes for this intermediate, TDP-4-keto-6-deoxyglucose 3, 5-epimerase (ep; $609 \mathrm{bp})$ and TDP-glucose 4-ketoreductase ( $k r ; 885 \mathrm{bp}$ ), were also cloned to the same vector to complete the 8,884 bp TDP-rhamnose biosynthesis system. A regiospecific flavonol 3-O-rhamnosyltransferase (ArGt-3) was cloned separately into pET32(a) + vector to generate pET32(a) + ArGt-3 because of the complications of 
restriction sites. The TDP-rhamnose sugar cassette along with pET32(a) + ArGt-3 were transformed into the $E$. coli BL21 (DE3) host for biotransformation reaction.

\section{Bioconversion of fisetin}

E. coli BL21 (DE3) harboring pIBR181-UGT78K1 (Strain $\mathrm{S}_{1}$ ) and pET32a $(+)$ ArGt-3 (Strain $\mathrm{S}_{5}$ ) were used to check the bioconversion of exogenously supplemented fisetin as described in materials and methods. The high performance liquid chromatography (HPLC) chromatograms of each extract from the biotransformation reactions showed new peaks at retention time $\sim 14.4 \mathrm{~min}$ for fisetin 3-O-glucoside and $\sim 14.8 \mathrm{~min}$ for fisetin 3-O-rhamnoside compared to the standard fisetin detected at $\sim 17.1 \mathrm{~min}$ at the UV absorbance of $320 \mathrm{~nm}$ (Figure 2). These product peaks detected from the glycosylation systems were further analyzed by high resolution liquid chromatographyquadrupole time-of-flight electrospray ionization mass spectrometry resolution (LC-QTOF-ESI/MS) in positive ion mode. The mass spectra displayed the exact mass of fisetin 3-O-rhamnoside $[\mathrm{M}+\mathrm{H}]^{+} m / z^{+} \sim 433.1096$ and fisetin 3-O-glucoside $[\mathrm{M}+\mathrm{H}]^{+} m / z^{+} \sim 449.1086$ along with the spectrum for fisetin $[\mathrm{M}+\mathrm{H}]^{+} m / z^{+} \sim 287.0564$ (Figure 2). The control experiments were carried out in identical conditions by supplementing fisetin in same strains without induction. In control strains, no bioconversion of fisetin was observed while analyzing HPLC-PDA.
To study the substrate inhibition for the biotransformation, different concentrations $(0.2,0.3,0.4,0.6,0.8,1.0 \mathrm{mM})$ of fisetin were fed into biocatalyses reaction systems (strains $\mathrm{S}_{1}$ and $\mathrm{S}_{5}$ ). The cell growth and substrate conversion were monitored in both shake flask cultures $(50 \mathrm{~mL})$ for $60 \mathrm{~h}$ as explained in materials and methods. The bioconversion of fisetin into its glycoside were exponentially increased until $48 \mathrm{~h}$ and become static for $60 \mathrm{~h}$. So, we calculated the total conversion based on $48 \mathrm{~h}$ incubation. The maximum bioconversion of fisetin recorded was $58.9 \%$ in strain $S_{1}$ and $41.8 \%$ in strain $S_{5}$ while supplementing $0.3 \mathrm{mM}$ of fisetin at the highest cellular growth of $\mathrm{OD}_{600} \sim 3.5$ for $48 \mathrm{~h}$. Upon increasing the concentration of fisetin from $0.3 \mathrm{mM}$, both strains showed relatively lower substrate conversion with decreased cell growth rates (Figure 3). The results suspected were toxic environment due to high concentration fisetin in E. coli culture medium [33].

\section{Glucose utilization and its impact on improved bioconversion by glucose facilitator protein}

Three different concentrations $(5,10,15 \%)$ of glucose were supplemented in separate cultures of strains $\mathrm{S}_{1}$ and $S_{5}$. The utilization of glucose by these strains was assessed by the biotransformation reactions of exogenously fed $0.3 \mathrm{mM}$ fisetin in HPLC-PDA. The HPLCPDA analysis found that $77.1 \%$ of fisetin was converted to fisetin-3-O-glucoside whereas the conversion of fisetin to fisetin 3-O-rhamnoside was limited to $48.5 \%$ in strains $\mathrm{S}_{1}$

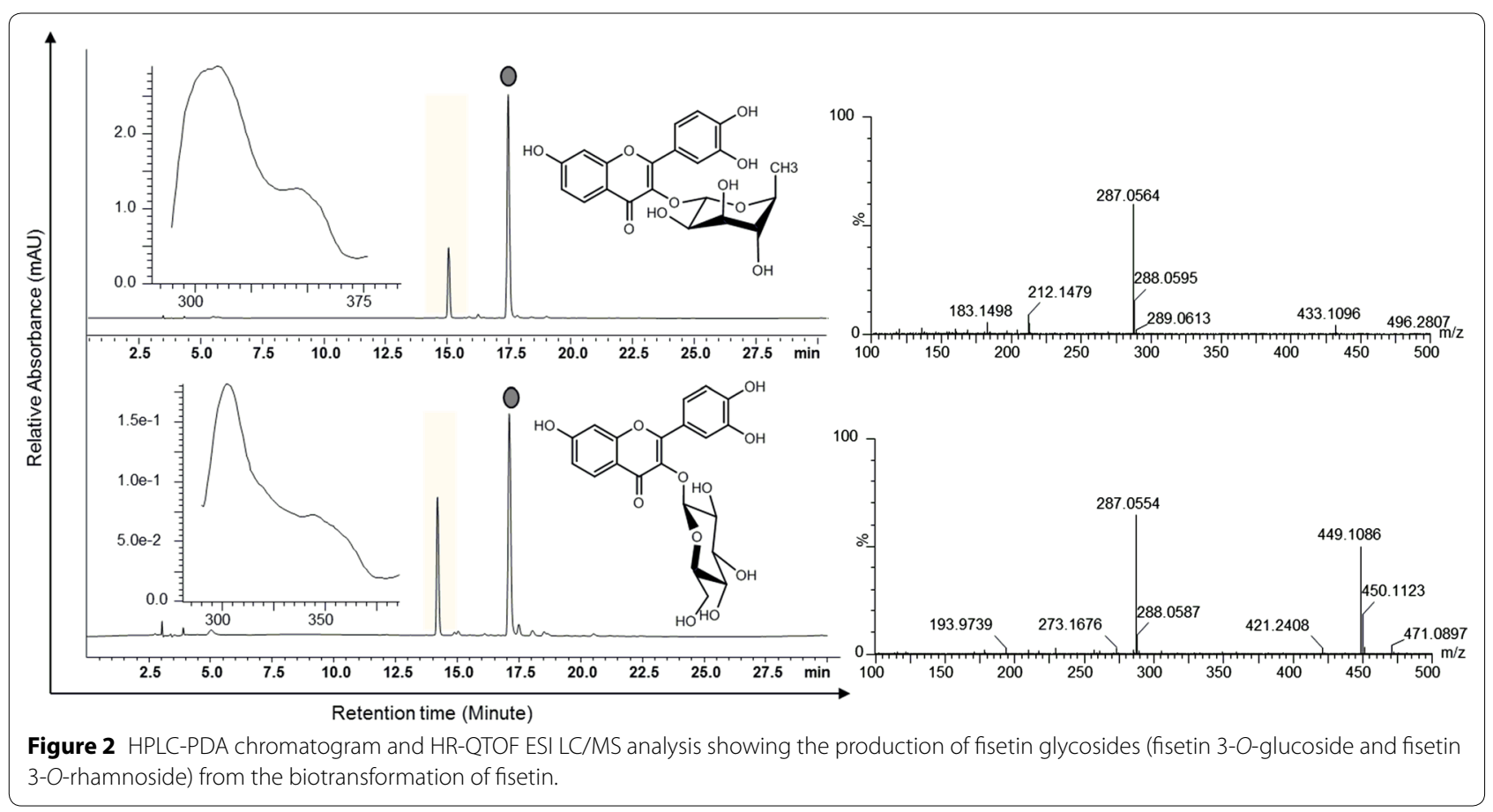




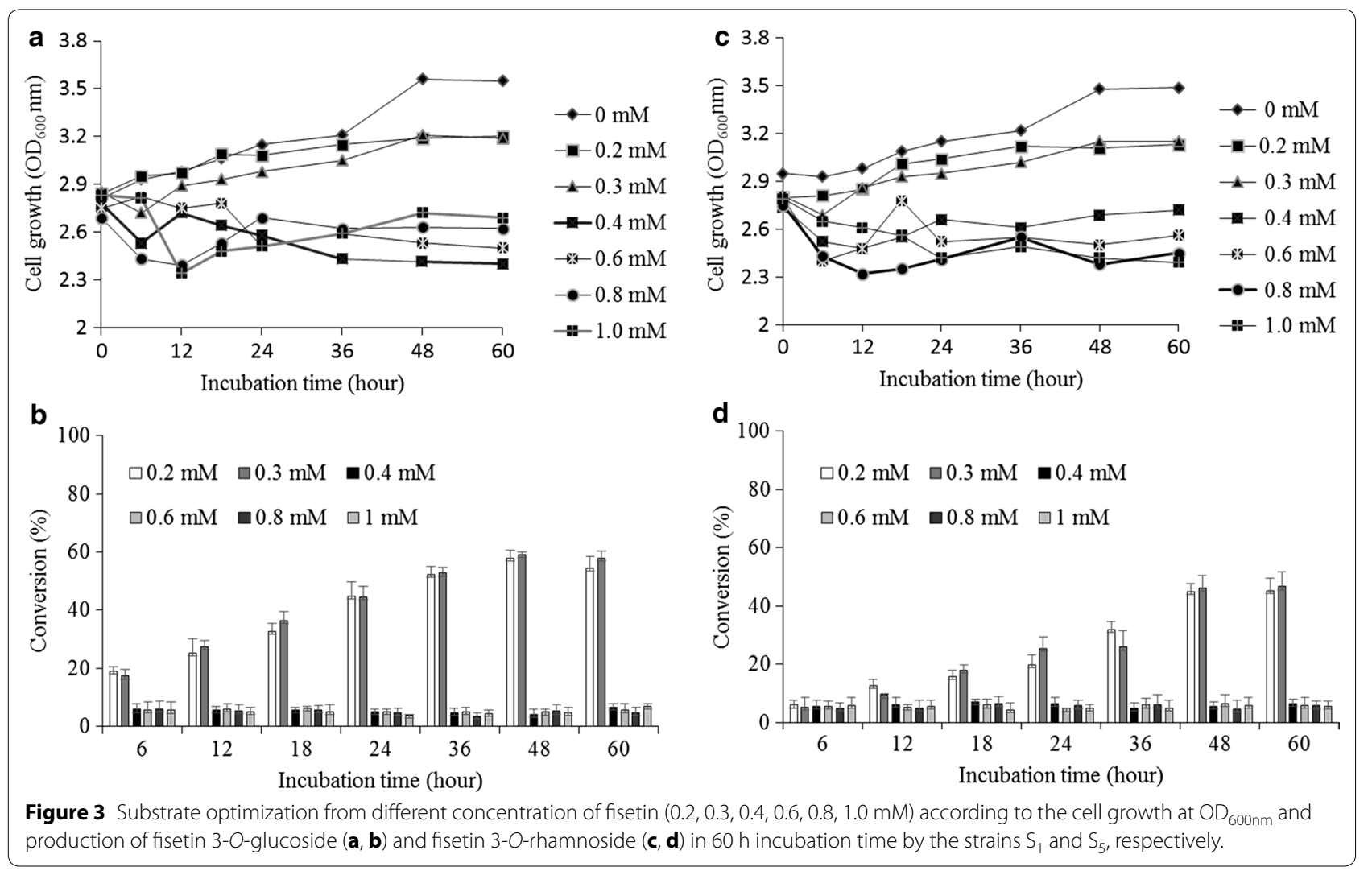

and $S_{5}$, respectively, upon addition of $10 \%$ additional glucose to the medium over $48 \mathrm{~h}$ of incubation (Figure $4 \mathrm{a}$ ). The supplementation of $10 \%$ glucose and $0.3 \mathrm{mM}$ fisetin acceptor substrate was optimized for utilization by the $E$. coli strains, balancing their metabolic flux and physiology in respect to the cellular growth and product formation.

The effects of supplementing extra $10 \%$ glucose in the medium and over expression of glucose facilitator protein on bioconversion of fisetin were also evaluated by feeding $0.3 \mathrm{mM}$ of fisetin in various strains. The recombinant strains harboring multi-vectors overexpressing UDP-glucose and TDP-rhamnose pathway specific genes but without glucose facilitator protein (strains $S_{2}$, $S_{6}$, and $S_{7}$ ), monocistronic vector carrying pathway specific genes without glucose facilitator protein (strains $S_{3}$, $\mathrm{S}_{8}$ ) and with glucose facilitator protein (strains $\mathrm{S}_{4}$ and $\mathrm{S}_{9}$ ), were grown in identical conditions for biotransformation. The samples were taken at different time intervals for HPLC-PDA analysis to determine the substrate conversion rate. Regarding 3-O-glucoside production, strain $\mathrm{S}_{3}$ (mono-cistronic vector construct carrying UDP-glucose biosynthesis genes) was found to be more effective $\left(\sim 85 \%\right.$ conversion) than strain $\mathrm{S}_{2}(\sim 80 \%$ conversion) which carried multiple vectors for the UDPglucose biosynthesis. Similar results were observed for the production of fisetin 3-O-rhamnoside. Strain $\mathrm{S}_{6}$ and $\mathrm{S}_{7}$ harboring TDP-rhamnose biosynthetic pathway genes in multiple vectors exhibited lower conversion $\left(\sim 68, \sim 72 \%\right.$, respectively) than strain $S_{8}(\sim 80 \%)$ in which those genes were cloned in mono-cistronic vector system. However, the recombinant strain $\mathrm{S}_{4}$ harboring a multi-monocistronic vector with sugar pathway genes for UDP-glucose glycosylation system along with glucose facilitator diffusion protein $(g l f)$ and $10 \%$ exogenous glucose supplement, it was able to convert $\sim 100 \%$ substrate into product within $48 \mathrm{~h}$. Similarly, for fisetin 3-O-rhamnoside production, the recombinant strain with multi-monocistronic TDP-rhamnose biosynthesis systems and facilitator proteins and 10\% exogenous glucose supplement, it $\left(\mathrm{S}_{9}\right)$ was able to convert $\sim 95 \%$ of fisetin (Figure 4b).

Thus, comparing the production profile of all the recombinant strains with multi-vector and multi-monocistronic NDP-sugar biosynthesis systems, use of the multi-monocistronic vector for multiple gene expression was found to be highly effective. With respect to the acceleration of the system, glucose facilitator protein could significantly increase the production of respective glycosides by $\sim 20 \%$ compared to strains without facilitator genes. 

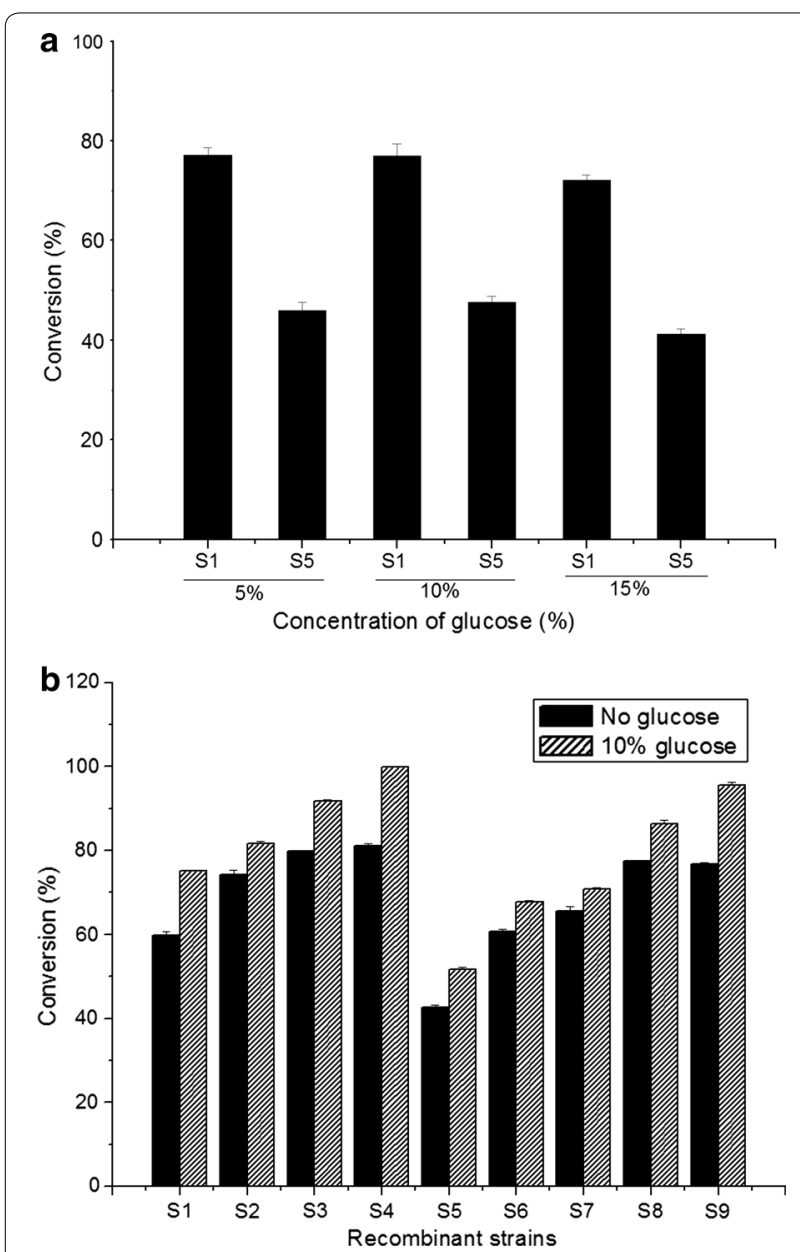

Figure 4 a Optimization of glucose concentration based on the recombinant strain $S_{1}$ and $S_{5}\left(S_{1}\right.$ and $S_{5}$ contains only GTs UGT78K1 and $\mathrm{ArGt}-3$, respectively) in $48 \mathrm{~h}$ incubation. Maximum conversion of fisetin to respective glycosides was achieved while supplementing $10 \%$ additional glucose in the medium. b Production profile of respective glycosides in all the constructed recombinant strains $\left(\mathrm{S}_{1}-\mathrm{S}_{9}\right)$ in optimized concentration of substrate $(0.3 \mathrm{mM})$ and glucose $(10 \%)$ in $48 \mathrm{~h}$ incubation time. Maximum conversion of fisetin to fisetin 3-O-glucoside was achieved to be $\sim 100 \%$. The conversion of fisetin to fisetin 3-O-rhamnoside was $~ 96 \%$.

\section{Scale-up by fermentation}

The recombinant strains $\mathrm{S}_{4}$ and $\mathrm{S}_{9}$ were chosen for large scale production of fisetin 3-O-glucoside and fisetin 3-O-rhamnoside respectively as both strains exhibited efficient bioconversion of fisetin (Figure 4b). Cultures of both strains were prepared for 3-L fermentation as described in materials and methods where optimized concentrations of substrate $(\sim 300 \mathrm{mg}$ in $3 \mathrm{~L}(\sim 0.35 \mathrm{mM}))$ and glucose $(10 \%)$ were supplemented. The temperature of the fermentor was kept at $25^{\circ} \mathrm{C}$ and the $\mathrm{pH}$ was 7.0 during the entire period for both systems. The culture medium were harvested at a regular time interval of $12 \mathrm{~h}$ and analyzed by HPLC-PDA to monitor the conversion of fisetin into its glycosides. The rest of the fermentation condition was identical to our previous studies [30, 34].

The HPLC analysis of the 3-L fermentor bioconversion samples at $12 \mathrm{~h}$ revealed complete conversion of fisetin to fisetin 3-O-glucoside and fisetin 3-O-rhamnoside by respective strains (Figure 5). Thus, additional $300 \mathrm{mg}$ of fisetin was added in $12 \mathrm{~h}$ and continued the fermentor under identical conditions. The HPLC samples analysis at $24 \mathrm{~h}$ also exhibited approximately $100 \%$ conversion of fisetin to glycosides. Hence, again the same amount of fisetin was added at $24 \mathrm{~h}$ and proceeded the fermentation. However, after $24 \mathrm{~h}$, the bioconversion of fisetin was slower than previous in both systems. The conversion rate was almost constant from 48 to 60 h (Figure 5). The fisetin added at $24 \mathrm{~h}$ was not completely converted until $60 \mathrm{~h}$. Overall calculations revealed the production of $\sim 1.178 \mathrm{~g}$ of fisetin $3-O$-glucoside $[\sim 393 \mathrm{mg} / \mathrm{L}$ $(\sim 0.30 \mathrm{mM})]$ and $1.026 \mathrm{~g}$ of fisetin 3-O-rhamnoside $[\sim 342 \mathrm{mg} / \mathrm{L}(\sim 0.27 \mathrm{mM})]$ in $48 \mathrm{~h}$ from the $3 \mathrm{~L}$ fermentor from $0.9 \mathrm{~g}(\sim 1.04 \mathrm{M})$ fisetin supplemented (Figure 5).

\section{Biotransformation of other flavonols}

After successfully probing and comparing the monocistronic and multivector UDP-glucose and TDP-rhamnose biosynthesis systems for the biotransformation of
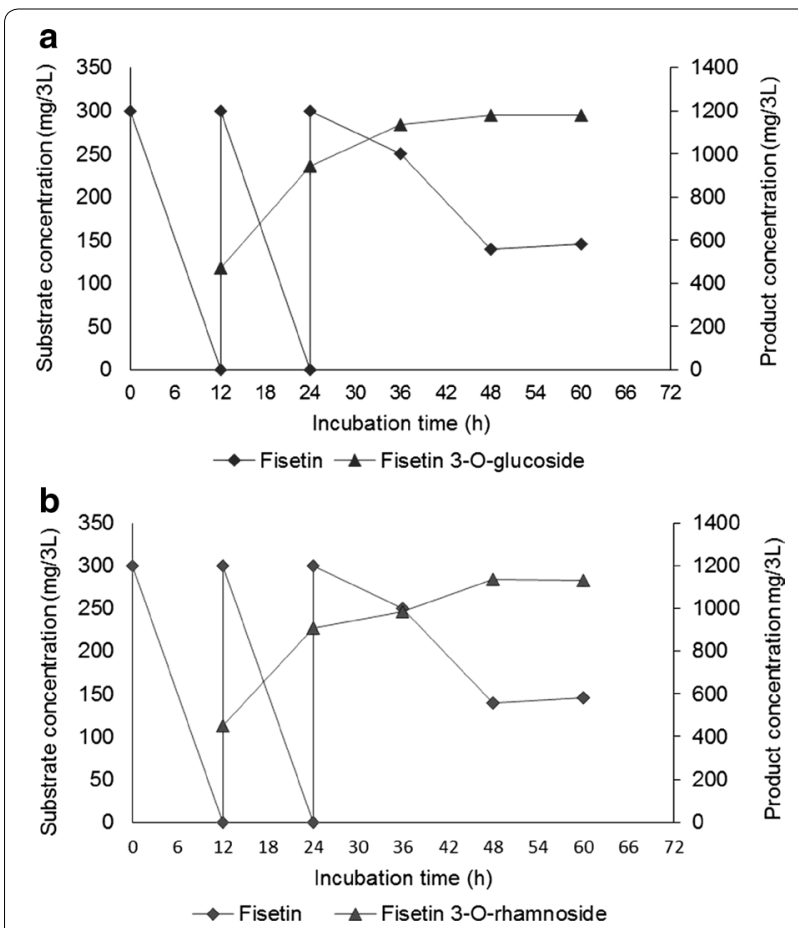

Figure $\mathbf{5}$ The scale-up production of fisetin glycosides in $3 \mathrm{~L}$ fermentation at different time intervals. a Production profile of fisetin 3-O-glucoside by strain $\mathrm{S}_{4}$. b Production profile of fisetin 3-O-rhamnoside by strain $\mathrm{S}_{9}$. $0.35 \mathrm{mM}$ fisetin dissolve in DMSO was successively added three times in both 3 - $L$ fermentation systems. 
fisetin as a substrate, we used the best monocistronic UDP-glucose and TDP-rhamnose biosynthesis genes and glucose facilitator gene containing systems (strains $\mathrm{S}_{4}$ and $\mathrm{S}_{9}$, respectively) to synthesize other flavonol glycosides. Kaempferol, myricetin, morin and quercetin were biotransformed into their respective glucosides and rhamnosides. The biotransformation of all the flavonols were done in a shake flask supplementing $10 \%$ glucose in culture medium along with $0.3 \mathrm{mM}$ of each individual flavonoid as substrate. Similar to the fisetin, there was approximately 95-100\% conversion of kaempferol, quercetin and myricetin substrates into their respective glucosides and rhamnosides. However, the conversion of morin was limited to $<40 \%$ in strain $\mathrm{S}_{4}$ and $<15 \%$ in strain $\mathrm{S}_{9}$ (Figure 6, Additional file 1: Figure S3). These regiospecific glucoside and rhamnoside products of other flavonols were confirmed by high resolution mass analyses (Additional file 1: Figure S4).

Thus, the overall result of the study indicates that the assembly of biological standard parts in multi-monocistronic fashion under promoters of the same strength is of great important for the production of bioactive flavonoid glycosides. This system yielded higher conversion rates than the multi-vector system reported previously. It is certain that production of the glycosides of flavonols other than morin should be similar to fisetin which we probed in the constructed system (Additional file 1: Figure S3).

\section{Discussion}

A multi-monocistronic operon system (piBR181) was generated previously modifying the commercially available vector $\mathrm{pET} 28 \mathrm{a}(+)$ in order to clone multiple genes

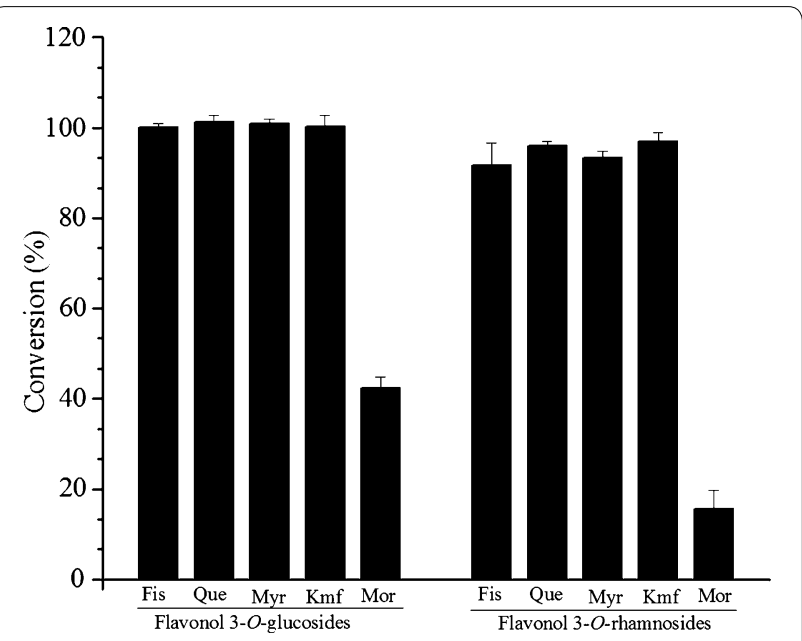

Figure 6 Biotransformation of other flavonols (Fis-fisetin, Quequercetin, Myr-myricetin, Kmf-kaempferol, Mor-morin) into respective glycosides using the best recombinant strains $\left(\mathrm{S}_{4}\right.$ and $\left.\mathrm{S}_{9}\right)$. under their own promoter [26]. The construct constitutes of computationally designed 181-bp ds-DNA bio-parts ligated to pET-28a (+) at the $B g l \mathrm{II}$ and $\mathrm{XhoI}$ restriction sites replacing original one to generate expression vector piBR181. However, BglII and BamHI-XhoI restriction sites presents before the promoter and after the transcription termination sites for multi-monocistronic operon assembly. Likewise, SpeI-HindIII remained was for PCR cloning site. Large number of gene can be cloned into this vector system and their functional expression are assumed to be independent transcriptome because they have independent bio-parts (T7 promoter, Lac operator, Ribosome binding site and T7 terminator). Having multiple copy number, it is applicable for multiple gene expression in multi-copy number transcriptome so that high yield production could be achieved. Moreover, necessity of using multiple vectors for heterologous protein expression and use of various antibiotic creates stress to the culture medium can be reduced. But the limitation is, five restriction sites (BglII, SpeI, HindIII, BamHI and $X h o I)$ must be absent from the gene sequence and vector itself.

The production of flavonoid was much higher in monocistronic operon compared to pseudo-operon where the distance between the start and end of the operon was an important factor in reducing premature transcription termination and mRNA degradation [35, 36]. Multiple circuits in an operon with the termination sequence present at every end of the gene set under the same promoter could therefore improve multi-round transcription by recycling the $T_{7}$ RNA polymerase quickly, thereby facilitating proper folding of multiple proteins [37]. In present study also, the efficiency of multi-monocistronic sugar biosynthesis system compared to multiple vector systems was found to be remarkable for producing flavonol glycosides. Each recombinant strains harboring multivectors and multi-monocistronic vector constructed using UDP-glucose and TDP-rhamnose biosynthesis pathway genes were compared for production of fisetin 3-O-glucoside and fisetin-3-O-rhamnoside catalyzed by UGT78K1 and ArGt-3 with the substrate fisetin. The production of flavonoid glycosides was comparably high using multi-monocistronic constructs in this experiment (Figure $4 \mathrm{~b}$ ). In previous studies, $24 \mathrm{mg} / \mathrm{L}$ of $3-O-r h a m-$ nosyl quercetin and $12.9 \mathrm{mg} / \mathrm{mL} 3-O$-rhamnosyl kaempferol was produced using a multiple vector system [31]. Likewise, The relative bioconversion of flavonols into rhamnosides using the same glycosyltransferases (ArGt-3), although $100 \%$ quercetin was converted into product while the other flavonols (kaempferol-85.7\%, Myricetin-20.5\%, Fisetin-62.3\% and morin-0\%) were converted by a lesser amount [38]. Similarly, Kim et al. [39] reported the maximum production of $150 \mathrm{mg} / \mathrm{L}$ of 
quercetin 3-O-rhmanoside as and $200 \mathrm{mg} / \mathrm{L}$ of kaempferol 3-O-rhamanoside. Similarly, $98 \mathrm{mg} / \mathrm{L}$ of quercetin 3-O-(6-deoxytalose) was produced through $E$. coli metabolic engineering approach [40].

Looking at the aforementioned production level of each flavonol glycosides, we could hypothesize several reasons to increase the titer more than previously reported. In most of the engineering strategy NDP-sugar biosynthesis pathway specific genes has been deleted to divert the metabolic flux for desirable sugar pool increment [31, $38,40,41]$. But this may require additional supplementation of carbon source and limits the optimum growth as compared to wild type strain. Similarly, use of multiple vectors can affect the production level since various antibiotics in culture broth limits the cell growth [35], different rate of plasmid replication because of varying plasmid origin [42] and multiple gene expression under different promoter strength can have varied expression of genes which could ultimately reduce the production of target compounds [14, 42]. Use of synthetic biology tools by engineering each regulatory components of an expression vector itself for the assembly of multiple genes and allow them to express consecutively could address aforementioned limitations of high yield production of target compounds from microbial cells [36, 43]. So there will be no metabolic burden for cell itself because of similar strength promoter architecture and could achieve tunable gene expression in transcriptional level. Use of single vector system in multi-monocistronic fashion and assembly of multi-pathway genes has proven advantages over old strategies of constructing cell factory with multiple antibiotic resistance vectors containing pathway specific genes $[44,45]$. Production of vaccine through construction of multi-gene expression cassette [46], tandem repetitive promoter in expression vector increases the expression level of genes ultimately scale up in secondary metabolite production [47], gram scale production of chondroitin [24] and catechin [25] using synthetic vectors in $E$. coli are the successful examples.

The construction of an artificial sugar cassette facilitated bacterial cell factory and subsequent re-direction of metabolic flux towards desirable products achieved significant production of flavonol 3-O-glucoside and 3-O-rhamnoside compared to previously reported data. However, further strengthening sugar biosynthesis system assembling glucose facilitator diffusion protein (Glf) with the goal of increasing the pool of intermediate product (glucose 1-phosphate) effectively increased the production of each glycoside by $20 \%$. Consequently, the production of fisetin 3-O-glucoside was approximately $1.178 \mathrm{~g} / 3 \mathrm{~L}$-fermentation $(\sim 393 \mathrm{mg} / \mathrm{L})$ in $48 \mathrm{~h}$ and $1.026 \mathrm{~g} / 3 \mathrm{~L}$-fermentation $(\sim 342 \mathrm{mg} / \mathrm{L})$ of fisetin 3-O-rhamnoside within the same time limit. Since the conversion to both glycosides was remarkably high, these are the best strains and production yields ever reported.

The same recombinant systems were also used to synthesize other regiospecific flavonol glycosides using kaempferol, quercetin, myricetin and morin as substrates. The result demonstrated a relative product conversion rates of $\sim 100 \%$ for quercetin, myricetin and kaempferol for 3-O-glucosides production while $~ 95 \%$ for quercetin, myricetin and kaempferol for 3-O-rhamnosides production in $48 \mathrm{~h}$ while supplementing $0.3 \mathrm{mM}$ of each substrate (Figure 6). Thus, the proposed glycosylation systems could be used as model systems in the glycoconjugation of similar derivatives as pharmaceutical ingredients.

\section{Methods}

\section{Microorganisms, plasmids, culture conditions and chemicals}

All the strains, vectors and plasmids used in this study are listed in Table 1. Recombinant strains harboring the NDP-sugar biosynthesis genes are numbered from $S_{1}$ to $\mathrm{S}_{9}$. For the selection and maintenance of plasmids, E. coli strains were grown at $37^{\circ} \mathrm{C}$ in Luria-Bertani (LB) broth or on agar plates supplemented with the appropriate amount of antibiotics (ampicillin-100 $\mu \mathrm{g} / \mathrm{mL}$, kanamycin-50 $\mu \mathrm{g} / \mathrm{mL}$, streptomycin $50 \mu \mathrm{g} / \mathrm{mL}$ and chloramphenicol $50 \mu \mathrm{g} / \mathrm{mL}$ ). pGEM-T ${ }^{\circ}$ easy vector (Madison, Promega), pET32a $(+)$, pCDF-Duet-1, pACYC-Duet-1 (Novagen, Darmstadt, Germany) and piBR181 vectors were used for the cloning of polymerase chain reaction (PCR) products and for expression of the genes (Additional file 1: Table S1). All the chemicals (methanol, water, ethyl acetate, etc.) used in this study were purchased from either Sigma-Aldrich or another high grade commercial source. All restriction enzymes used in the cloning process were obtained from Takara (Shiga, Japan). Oligonucleotide primers were from Genotech (Daejeon, Korea). The rest of the chemicals purchased from commercially available sources were of high grade.

\section{Nucleic acid manipulation and construction of vectors}

All the sugar pathway genes were overexpressed into E. coli BL21 (DE3) for specific sugar cassettes construction. To enhance the cytoplasmic pool of UDP-glucose in cell cytosol, the genes for glucokinase ( $g l k-Z y m o m o n a s$ mobilisi, GenBank accession no. AE008692.2), phosphoglucomutase (pgm2-Bacillus licheniformis DSM 13, GenBank accession no. YP_006712377.1), glucose 1-phosphate uridylyltransferase (galU-E. coli K-12, GenBank accession no. CP001509.3) and glucosyltransferases (UGT78K1-G. $\max$ (L) Merr. (Black Soybean), GenBank accession no. ADC96620.1) including a glucose facilitator diffusion protein $(g l f-Z$. mobilisi, GenBank accession no. 
Table 1 Bacterial strains, plasmids used in this study

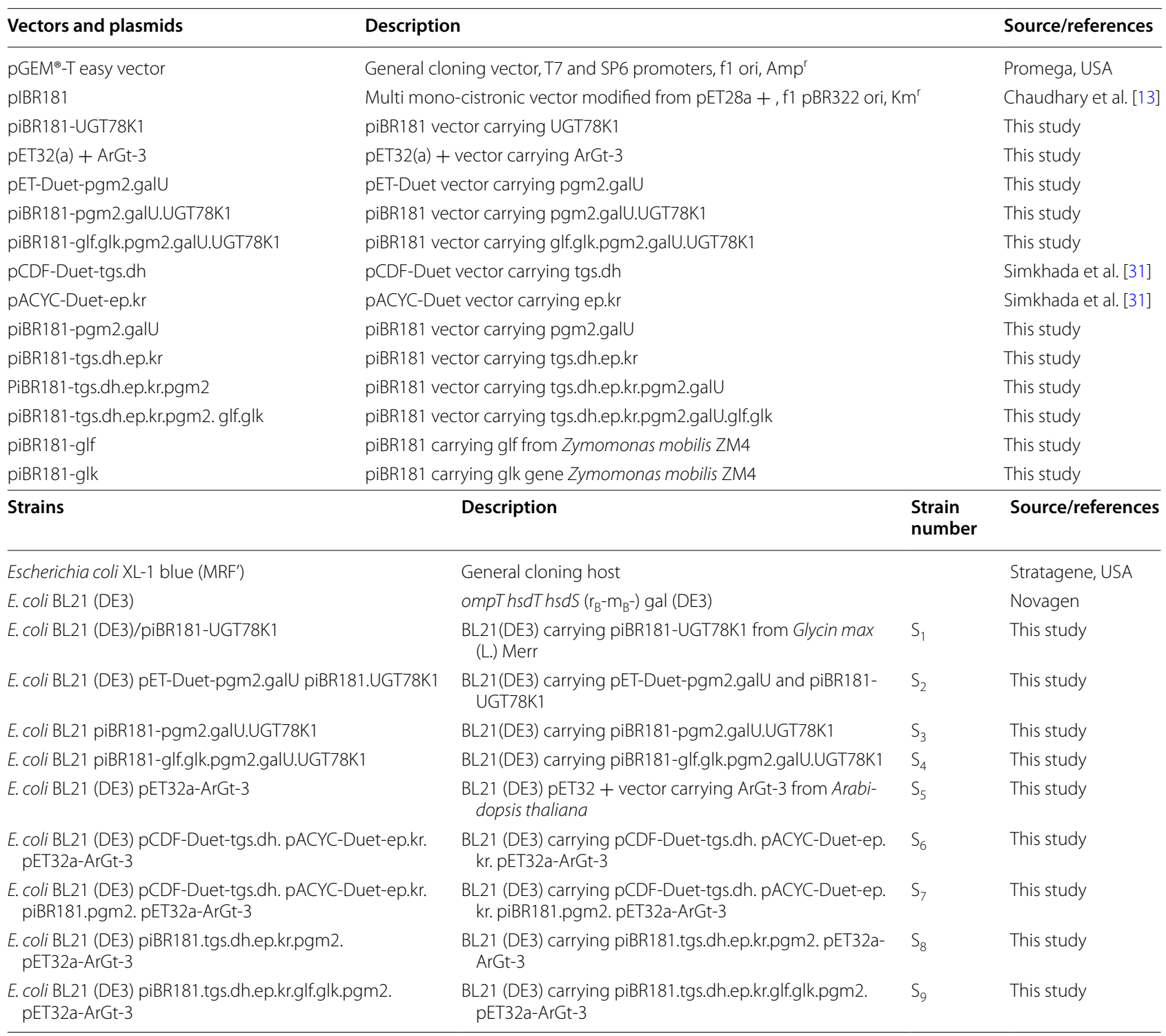

AE008692.2) provided by Dr. Stephanie Bringer-Meyer (Institute of Bio- and Geoscience, Germany) [32] were cloned in pIBR181 vector to construct the glucose cassette containing the glucosylation system. $g l f, g l k, p g m 2$ including glucose 1-phosphate thymidylyltransferase (tgs-E. coli K-12, GenBank accession no. YP_490281.1), TDP-glucose 4, 6-dehydratase (dh-Salmonella enterica LT2, GenBank accession no. NC_003197.1), TDP4-keto-6-deoxyglucose 3, 5-epimerase and TDP-glucose 4-ketoreductase (ep and kr-Streptomyces antibioticus Tu99 with GenBank AAF59933.1 and AF237894.1, respectively) were cloned in the same pIBR181 vector under individual T7 promoter. Using the primer pairs of $X b a \mathrm{I}$ 'F' and HindIII 'R', all the genes involved in
UDP-glucose and TDP-rhamnose cassettes were PCR amplified from their respective sources and manipulated into the $\mathrm{pGEM}^{\ominus}-\mathrm{T}$ easy vector. The nucleotide sequences were later confirmed by sequencing. Three genes $d h, e p$ and $k r$ were PCR amplified from the previously reported vector constructs [31] for cloning in pIBR181. Finally, the sugar pathway genes, including glucosyltransferase (UGT78K1), containing terminal XbaI/HindIII restriction sites were individually cloned into the endonuclease digested vector piBR181 at SpeI/HindIII sites for the final construct. The vector piBR181 is a genetic circuit containing 181-bp double stranded DNA and includes computationally designed bio-parts between the BglII and XhoI restriction site and the SpeI/HindIII cloning 
site for single ligation. BamHI/XhoI restriction sites are located before the promoter and after the transcription termination to facilitate multiple assemblies of the sugar pathway genes [26]. Unfortunately, rhamnosyltransferase (ArGt-3, A. thaliana, GenBank accession no. AF360160) [31] could not be cloned into piBR181 because of the five enzymes restriction. So, this glycosyltransferase was cloned into pET32a(+) vector in EcoRI/XhoI sites under the same promoter (T7) and plasmid copy number as pIBR181.

\section{Substrate inhibition and optimization}

Bacterial strains harboring only GTs, $S_{1}$ and $S_{5}$ of the same cell density were used for the substrate inhibition test. Different concentrations of fisetin $(0.2 \mathrm{mM}, 0.3 \mathrm{mM}$, $0.4 \mu \mathrm{M}, 0.6 \mathrm{mM}, 0.8 \mathrm{mM}, 1.0 \mathrm{mM}$ ) dissolved in dimethylsulfoxide (DMSO) was used to bio-transform in fed batch $50 \mathrm{~mL}$ culture volume. At equal incubation time intervals (i.e. $6 \mathrm{~h}$ intervals for $24 \mathrm{~h}$ and $12 \mathrm{~h}$ intervals for $60 \mathrm{~h}$ ) but the data was considered of $48 \mathrm{~h}$ for analyses, $1 \mathrm{~mL}$ samples were taken from each flask and centrifuged at $12,000 \mathrm{rpm}$ for $10 \mathrm{~min}$. The supernatants were extracted using a double volume of ethyl acetate while the cell pellets were re-suspended in water and the cell densities measured by spectrophotometer at $600 \mathrm{~nm}$ ultraviolet (UV) absorbance. The ethyl acetate fractions were dried and re-suspended into $100 \mu \mathrm{L}$ methanol and subjected to photo diode array connected high performance liquid chromatography (HPLC-PDA) analyses. The peak area was used to calculate the total conversion of substrate into glycoside product over cell growth in reference to the standard curve of fisetin.

\section{Glucose supplement and optimization}

Recombinant strains $S_{1}$ and $S_{5}$ were also considered for glucose supplementation and optimization in a biotransformation reaction. These strains were grown under identical conditions, keeping E. coli BL21 (DE3) as a control strain for growth measurement. The samples used for HPLC-PDA analysis and cell density measurement were prepared as mentioned above. Optimized fisetin substrate concentration $(0.3 \mathrm{mM})$ was supplemented in the cultures with three different concentrations of glucose (5, 10 and $15 \%)$ to determine the optimum glucose concentration for biotransformation. Finally, HPLC-PDA analysis was carried out and conversion of the substrate into product was verified over incubation time intervals according to the cell growth.

\section{Bioconversion and Scale-up whole-cell biotransformation}

E. coli strains $\mathrm{S}_{1}$ and $\mathrm{S}_{5}$ were used for whole cell biocatalysis assay. These strains were grown on LB medium supplemented with kanamycin $(50 \mu \mathrm{g} / \mathrm{mL})$ and ampicillin $(100 \mu \mathrm{g} / \mathrm{mL})$ antibiotics, respectively. The cultures were induced with $0.5 \mathrm{mM}$ isopropyl-1-thio$\beta$-D-galactopyranoside (IPTG) when the cells' $\mathrm{OD}_{600 \mathrm{~nm}}$ reached $0.5-0.7$ while incubating at $37^{\circ} \mathrm{C}$. The cultures were incubated at $20^{\circ} \mathrm{C}$ for $20 \mathrm{~h}$ for functional expression of protein. After $20 \mathrm{~h}$ of incubation at $20^{\circ} \mathrm{C}$, cultures were fed with $0.3 \mathrm{mM}$ fisetin and incubated for $48 \mathrm{~h}$ to produce fisetin-3-O-glucoside and fisetin 3-O-rhamnoside from the respective strains. Identical conditions were used for comparison of the biotransformation of fisetin using other strains $\left(\mathrm{S}_{1}-\mathrm{S}_{9}\right)$ to determine the best strain for large-scale production of flavonol glycosides. The biotransformation of other flavonols (quercetin, kaempferol, myricetin, and morin) was also carried out under identical conditions.

For the lab scale biotransformation of fisetin in $3 \mathrm{~L}$ fermentor using the best optimized strains $\left(\mathrm{S}_{4}\right.$ and $\left.\mathrm{S}_{9}\right)$, identical conditions of $\mathrm{pH}$, temperature $\left(25^{\circ} \mathrm{C}\right)$ and dissolved oxygen were used as reported previously [30]. Sterile glucose (10\% final concentration) was supplemented to the fermentor at every $1 \mathrm{~h}$ intervals after addition of fisetin to the induced cultures up to $36 \mathrm{~h}$. The samples were taken at every $12 \mathrm{~h}$ intervals for the HPLC-PDA analysis up to $60 \mathrm{~h}$ after initial addition of fisetin ( $300 \mathrm{mg}-0.35 \mathrm{mM}$ ) to the grown and induced cultures of $\mathrm{S}_{4}$ and $\mathrm{S}_{9}$. The same amount of fisetin was added after $12 \mathrm{~h}$ and $24 \mathrm{~h}$ of first addition of fisetin as it was completely converted at $12 \mathrm{~h}$ intervals.

\section{Product analysis and quantification}

The culture extracts dissolved in methanol were directly analyzed by reverse-phase HPLC-PDA connected with $\mathrm{C}_{18}$ column (Mightysil RP-18 GP $(4.6 \times 250 \mathrm{~mm}, 5 \mu \mathrm{m})$ at $320 \mathrm{~nm}$ using binary conditions of $\mathrm{H}_{2} \mathrm{O}(0.05 \%$ trifluroacetic acid buffer) and $100 \%$ acetonitrile $(\mathrm{ACN})$ at a flow rate of $1 \mathrm{ml} / \mathrm{min}$ for $30 \mathrm{~min}$. The ACN concentrations were: $10 \%$ (0-2 $\mathrm{min}), 70 \%(20-24 \mathrm{~min}), 100 \%$ (24-28 $\mathrm{min})$, and 50\% (28-30). For quantification of flavonoids, a calibration curve of authentic fisetin was created using 10, 25, 50, 100 and $200 \mu \mathrm{g} / \mathrm{mL}$ concentrations. The exact mass of the compounds were analyzed by liquid chromatography quadrupole time-of-flight electrospray ionization mass spectrometry LC-QTOF-ESI/ MS [ACQUITY (UPLC, Waters, Mil-ford, MA)-SYNAPT G2-S (Waters)] in the positive ion mode.

\section{Additional files}

Additional file 1: Figures S1. Construction of a recombinant glucose cassette using piBR181 mono-cistronic vector. Figure S2. Construction of an efficient rhamnosylation system using piBR181 mono-cistronic vector. The figure showing vector map containing cloning sites in a single circuit. Figure S3. HPLC-PDA analyses of other flavonols (kaempferol, myricetin, quercetin and morin). Figure S4. High resolution mass analyses confirmed flavonol glycosides. 


\section{Abbreviations}

TDP: thymidine diphosphate; UDP: uridine diphosphate; NDP: nucleotide diphosphate; HPLC: high performance liquid chromatography; PDA: photo diode array; LC: liquid chromatography; QTOF: quadrupole time-of-flight; ESI/ MS: electrospray ionization mass spectrometry; DMSO: dimethyl sulfoxide; IPTG: isopropyl $\beta$-D-1-thiogalactopyranoside; ACN: acetonitrile; TFA: trifluroacetic acid.

\section{Authors' contributions}

PP and RPP performed the majority of the experimental work and wrote the majority of the manuscript. SJK, RPP and PP were responsible for the original concept and supervised the work. NTHT did the majority of the molecular cloning work. AKC provided the concept of the vector and cloning multiple genes. All authors have read and approved the manuscript.

\section{Acknowledgements}

This work was supported by a Grant from the Next-Generation BioGreen 21 Program (SSAC, Grant\#: PJ011119012015), Rural Development Administration, Republic of Korea.

\section{Compliance with ethical guidelines}

\section{Competing interests}

The authors declare that they have no competing interests.

Received: 2 March 2015 Accepted: 11 May 2015

Published online: 09 June 2015

\section{References}

1. Xiao J, Muzashvili TS, Georgiev MI (2014) Advances in the biotechnological glycosylation of valuable flavonoids. Biotechnol Adv 32:1145-1156

2. Mouri C, Mozaffarian V, Zhang X, Laursen R (2014) Characterization of flavonols in plants used for textile dyeing and the significance of flavonol conjugates. Dyes Pigm 100:135-141

3. Perez-Vizcaino F, Duarte J (2010) Flavonols and cardiovascular disease. Mol Aspects Med 31:478-494

4. Kim H, Bartley GE, Arvik T, Lipson R, Nah SY, Seo K, Yokoyama W (2014) Dietary supplementation of chardonnay grape seed flour reduces plasma cholesterol concentration, hepatic steatosis, and abdominal fat content in high-fat diet-induced obese hamsters. J Agric Food Chem 62:1919-1925

5. Escriche I, Kadar M, Juan-Borrás M, Domenech E (2014) Suitability of antioxidant capacity flavonoids and phenolic acids for floral authentication of honey. Impact of industrial thermal treatment. Food Chem 142:135-143

6. Liu YJ, Zhan J, Liu XL, Wang Y, Jia J, He QQ (2014) Dietary flavonoids intake and risk of type 2 diabetes: a meta-analysis of prospective cohort studies. Clin Nutr 33:59-63

7. Liao XL, Luo JG, Kong LY (2013) Flavonoids from Millettia nitida var. Hirsutissima with their anti-coagulative activities and inhibitory effects on NO production. J Nat Med 67:856-861

8. Wang $X$ (2009) Structure, mechanism and engineering of plant natural product glycosyltransferases. FEBS Lett 583:3303-3309

9. Bartmańska A, Tronina T, Popłoński J, Huszcza E (2013) Biotransformation's of prenylated hop flavonoids for drug discovery and production. Curr Drug Metab 14:1083-1097

10. Wang A, Zhang F, Huang L, Yin X, Li H, Wang Q, Zeng Z, Xie T (2010) New progress in biocatalysis and biotransformation of flavonoids. J Med Plants Res 4:847-856

11. Kamionka M (2011) Engineering of therapeutic proteins production in Escherichia coli. Curr Pharm Biotechnol 12:268-274

12. Chen X, Zhou L, Tian K, Kumar A, Singh S, Prior BA, Wang Z (2013) Metabolic engineering of Escherichia coli: a sustainable industrial platform for bio-based chemical production. Biotechnol Adv 31:1200-1223

13. Fujii T, Narikawa T, Sumisa F, Arisawa A, Takeda K, Kato J (2006) Production of alpha, omega-alkanediols using Escherichia coli expressing a cytochrome P450 from Acinetobacter OC4. Biosci Biotechnol Biochem 70:1279-1285
14. Lee SK, Chou H, Ham TS, Lee TS, Keasling JD (2008) Metabolic engineering of microorganisms for biofuels production:from bugs to synthetic biology to fuels. Curr Opin Biotechnol 19:556-563

15. Alper $H$, Stephanopoulos $G$ (2009) Engineering for biofuels: exploiting innate microbial capacity or importing biosynthetic potential. Nat Rev Microbiol 10:715-723

16. Kwiecien I, Szopa A, Madej K, Ekjert H (2013) Arbutin production via biotransformation of hydroquinone in in vitro cultures of aromia melanocarpa (Michx) Elliott. Acta Biochim Pol 60:865-870

17. Yende SR, Harle UN, Chugule BB (2014) Therapeutic potential and health benefits of Sargassum species. Pharmacogn Rev 15:1-7

18. Alonso-Gutierrez J, Chan R, Batth TS, Adams PD, Keasling JD, Petzold CJ, Lee TS (2013) Metabolic engineering of Escherichia coli for limonene and perillyl alcohol production. Metab Eng 19:33-41

19. Lim CG, Fowler ZL, Hueller T, Schaffer S, Koffas MA (2011) High-yield resveratrol production in engineered Escherichia coli. Appl Environ Microbiol 77:3451-3460

20. Negrete A, Ng WI, Shiloach J (2010) Glucose uptake regulation in E. coli by the small RNA SgrS: comparative analysis of E. coli K-12 (JM109 and MG 1655) and E. coli B (BL21). Microb Cell Fact 9:75

21. Shiloach J, Rinas $\cup$ (2009) Glucose and acetate metabolism in E. coliSystem level analysis and biotechnological applications in protein production process. In: Lee SY (ed) System biology and biotechnology of Escherichia coli. Springer Science + Business Media B.V., USA pp 377-400

22. Miyahisa I, Funa N, Ohnishi Y, Martens S, Moriguchi T (2006) Sueharu: combinatorial biosynthesis of flavones and flavonols in Escherichia coli. Appl Microbiol Biotechnol 71:53-58

23. Horinouchi S (2009) Combinatorial biosynthesis of plant medicinal polyketides by microorganisms 13:197-204

24. He W, Fu L, Li G, Andrew Jones J, Linhardt RJ, Koffas M (2015) Production of chondroitin in metabolically engineered E. coli. Metab Eng 27:92-100

25. Zhao S, Jones JA, Lachance DM, Bhan N, Khalidi O, Vankataraman S, Wang Z, Koffas MA (2014) Improvement of catechin production in Escherichia coli through combinatorial metabolic engineering. Metab Eng 28C:43-53

26. Chaudhary AK, Park JW, Yoon YJ, Kim BG, Sohng JK (2013) Re-engineering of genetic circuit for 2-deoxystreptamine (2-DOS) biosynthesis in Escherichia coli BL21 (DE3). Biotechnol Lett 35:285-293

27. Sichwart S, Hetzler S, Broker D, Steinbuchel A (2011) Extension of the substrate utilization range of Ralstonia eutropha strain $\mathrm{H} 16$ by metabolic engineering to include mannose and glucose. Appl Environ Microbiol 77:1325-1334

28. Tozakidis IE, Sichwart S, Teese MG, Jose J (2014) Autotransporter mediated esterase display on Zymomonas mobilis and Zymobacter palmae. J Biotechnol 191:228-235

29. Jones P, Messner B, Nakajima J, Schaffner AR, Saito K (2003) UGT73C6 and UGT78D1, glycosyltransferases involved in flavonol glycoside biosynthesis in Arabidopsis thaliana. J Biol Chem 278:43910-43918

30. Pandey RP, Malla S, Simkhada D, Kim BG, Sohng JK (2013) Production of 3-O-xylosyl quercetin in Escherichia coli. Appl Microbiol Biotechnol 97:1889-1901

31. Simkhada D, Lee HC, Sohng JK (2010) Genetic engineering approach for the production of rhamnosyl and allosyl flavonoids from Escherichia coli. Biotechnol Bioeng 107:154-162

32. Siedler S, Bringer S, Blank LM, Bott M (2012) Engineering yield and rate of reductive biotransformation in Escherichia coli by partial cyclization of the pentose phosphate pathway and PTS-independent glucose transport. Appl Microbiol Biotechnol 93:1459-1467

33. Gabor M, Eperjessy E (1966) Antibacterial effect of fisetin and fisetidin. Nature 212:1273

34. Malla S, Pandey RP, Kim BG, Sohng JK (2013) Regiospecific modifications of naringenin for astragalin production in Escherichia coli. Biotechnol Bioeng 110:1889-1901

35. Lim HN, Lee Y, Hussein R (2011) Fundamental relationship between operon organization and gene expression. Proc Natl Acad Sci USA 108:10626-10631

36. Xu P, Vansiri A, Bhan N, Koffas MA (2012) ePathBrick: a synthetic biology platform for engineering metabolic pathways in E. coli. ACS Synth Biol 1:256-266

37. Ferrari R, Rivetti C, Dieci G (2004) Transcription reinitiation properties of bacteriophage $T_{7}$ RNA polymerase. Biochem Biophys Res Commun 315:376-380 
38. Thuan NH, Pandey RP, Thuy TT, Park JW, Sohng JK (2013) Improvement of region-specific production of myricetin-3-O- $\alpha$-L-rhamnoside in engineered Escherichia coli. Appl Biochem Biotechnol 171:1956-1967

39. Kim BG, Kim HJ, Ahn JH (2012) Production of bioactive flavonol rhamnosides by expression of plant genes in Escherichia coli. J Agric Food Chem 44:11143-11148

40. Yoon JA, Kim BG, Lee WJ, Lim Y, Chong Y, Ahn JH (2012) Production of a novel quercetin glycoside through metabolic engineering of Escherichia coli. Appl Environ Microbiol 78:4256-4262

41. Mao Z, Shin HD, Chen RR (2006) Engineering the E. coli UDP-glucose synthesis pathway for oligosaccharide synthesis. Biotechnol Prog 22:369-374

42. Aw R, Polizzi MK (2013) Can too many copies spoil broth? Microb Cell Fact $12: 128$

43. Xu P, Koffas MA (2013) Assembly of multi-gene pathways and combinatorial pathway libraries through ePathBrick vectors. Methods Mol Biol 1073:107-129
44. Na D, Kim TY, Lee SY (2010) Construction and optimization of synthetic pathways in metabolic engineering. Curr Opin Microbiol 13:363-370

45. Stephanopoulos G (2012) Synthetic biology and metabolic engineering. ACS Synth Biol 1:514-525

46. Du S, Li C, Wang Y, Liu C, Ren D, Li Y et al (2012) Construction and evaluation of a new triple-gene expression cassette vaccinia virus shuttle vector. J Virol Methods 185:175-183

47. Li M, Wang J, Geng Y, Wang Q, Qi Q (2012) A strategy of gene overexpression based on tandem repetitive promoters in Escherichia coli. Microb Cell Fact 11:19

\section{Submit your next manuscript to BioMed Central and take full advantage of:}

- Convenient online submission

- Thorough peer review

- No space constraints or color figure charges

- Immediate publication on acceptance

- Inclusion in PubMed, CAS, Scopus and Google Scholar

- Research which is freely available for redistribution

Submit your manuscript at

www.biomedcentral.com/submit

C Biomed Central 